Article

\title{
Effects of Early Intervention with Antibiotics and Maternal Fecal Microbiota on Transcriptomic Profiling Ileal Mucusa in Neonatal Pigs
}

\author{
Rongying $\mathrm{Xu}^{1,2}$, Jiajia Wan ${ }^{1,2}$, Chunhui Lin ${ }^{1,2}$ and Yong $\mathrm{Su}^{1,2, *}$ \\ 1 Laboratory of Gastrointestinal Microbiology, Jiangsu Key Laboratory of Gastrointestinal Nutrition and \\ Animal Health, College of Animal Science and Technology, Nanjing Agricultural University, Nanjing 210095, \\ China; 2017105053@njau.edu.cn (R.X.); 2016105047@njau.edu.cn (J.W.); 2016105048@njau.edu.cn (C.L.) \\ 2 National Center for International Research on Animal Gut Nutrition, Nanjing Agricultural University, \\ Nanjing 210095, China \\ * Correspondence: yong.su@njau.edu.cn; Tel.: +86-2584-395-523
}

Received: 29 November 2019; Accepted: 13 January 2020; Published: 18 January 2020

\begin{abstract}
This study aimed to investigate the effects of early intervention with antibiotics and maternal fecal microbiota on ileal morphology and barrier function, and transcriptomic profiling in neonatal piglets. Piglets in the amoxicillin (AM), fecal microbiota transplantation (FMT), and control (CO) groups were orally administrated with amoxicillin solution $(6.94 \mathrm{mg} / \mathrm{mL})$, maternal fecal microbiota suspension $\left[>10^{9}\right.$ colony forming unit $\left.(\mathrm{CFU}) / \mathrm{mL}\right]$, and physiological saline, respectively. Compared with the CO group, early intervention with AM or FMT significantly decreased ileal crypt depth on day 7 and altered gene expression profiles in ileum on days 7 and 21, and especially promoted the expression of chemokines (CCL5, CXCL9, and CXCL11) involved in the toll-like receptor signaling pathway on day 21. FMT changed major immune activities from B cell immunity on day 7 to $T$ cell immunity on day 21 in the ileum. On the other hand, both AM and FMT predominantly downregulated the gene expression of toll-like receptor 4 (TLR4). In summary, both early interventions modulated intestinal barrier function and immune system in the ileum with a low impact on ileal morphology and development.
\end{abstract}

Keywords: antibiotic; fecal microbiota transplantation; ileal mucosa; neonatal pig; transcriptomic profiling

\section{Introduction}

The early colonization of the gut microbiota, which is considered to be the major antigen challenge for the newborn, is essential for the maturation of the gut-associated lymphoid tissue and for the developmental regulation of the intestinal physiology [1-3]. In addition, on molecular levels, members of intestinal microbiota have been reported to have a great capacity to regulate the expression of host genes related to mucosal barrier function and a variety of other intestinal functions, including nutrient absorption, metabolism, angiogenesis, and intestinal maturation [4]. During early life stages, intestinal microbiota is dynamic and can be easily influenced by environmental conditions [5-7]; therefore, modulating the intestinal microbiota development in early life as a strategy to maintain host health in later life has drawn wide attention.

Antibiotics can cause changes in the normal development of intestinal microbiota, generally coinciding with a decrease in phylogenetic diversity [8-10]. Amoxicillin, a frequently used antibiotic, produced simplified hindgut bacteria communities with decreased counts in mice [11], and reduced the numbers of gut microbiota diversity in rats [12]. Amoxicillin treatment fundamentally eliminates Lactobacillus spp. in the small intestine [13] and these microbiota changes reduce the expression of 
major histocompatibility complex (MHC) class I and II genes in the small and large intestine in suckling rats [14]. However, the impact of early intervention with antibiotics on intestinal function in neonatal pigs is not fully clear.

Another modulation strategy is fecal microbiota transplantation (FMT), which can normalize the composition and functionality of gut microbiota [15]. It refers to the process of transplanting the functional flora of donor feces into the gastrointestinal tract of the recipient and reconstructing new intestinal microbiota, which is mainly used in humans [16]. Early FMT treatment not only induced changes in offspring's gut microbiota composition (mostly in the ileum), but also altered the abundances of predicted bacterial pathways, affected intestinal morphology, and modulated duodenal gene expression in newborn pigs [17]. Taken together, FMT demonstrates an extensive impact on early-life intestinal microbiota and host phenotype is changed accordingly. Maternal fecal microbiota, as an environmental factor, inevitably comes into contact with neonate at birth. Our previous study suggested maternal fecal microbiota may play an important role in the process of gut microbiota colonization in piglets [18]; thus, this early intervention may further impact the intestinal development and function of neonatal pigs.

In the present study, oral administration with amoxicillin or maternal fecal microbiota was performed in an early intervention model on pig gut microbiota. Although our previous study investigated short- and long-term effects of early intervention with amoxicillin and maternal fecal microbiota on intestinal microbiota and metabolites in newborn piglets [19], the corresponding impact on intestinal function is yet unclear. Therefore, the aim of this study was to investigate the effect of early oral administration of amoxicillin and maternal fecal microbiota transplantation on the ileal mucosa gene expression and intestinal function in neonatal piglets.

\section{Materials and Methods}

\subsection{Ethics Statement}

The present study followed the guidelines for animal care and use of Nanjing Agricultural University (Nanjing, Jiangsu province, China) and the whole experiment process was under the support of the Animal Care and Use Committee (SYXK2017-0027).

\subsection{Donor Material Preparation}

The preparation of maternal fecal microbiota suspension was adapted from a previous study [20]. Briefly, mixing fresh fecal samples from candidate pregnant sows with anaerobic sterile $0.9 \% \mathrm{NaCl}$ solution (1:5) and sterile filtered. The obtained filtrate was centrifuged (2000 rpm, $10 \mathrm{~min}$ ) and then the supernatant was dispensed to $10 \mathrm{ml}$ sterile tubes and frozen at $-80{ }^{\circ} \mathrm{C}$. The entire preparation process was under anaerobic condition.

\subsection{Animal Experiment and Sampling}

Five litters of healthy neonatal 0-day-old piglets (Duroc $\times$ Landrace $\times$ Yorkshire, nine piglets in each litter) were used in this study. Each litter was randomly allocated into the CO, AM, or FMT groups, with three piglets in each group. On days 1-6, piglets in the maternal fecal microbiota transplantation (FMT) group were orally administered with $3 \mathrm{ml}$ fecal microbiota suspension $\left[>10^{9}\right.$ colony forming unit $(\mathrm{CFU}) / \mathrm{mL}]$ at 8:00 am every day, piglets in the amoxicillin treatment (AM) group and the control (CO) group were orally supplemented with the same volume of amoxicillin $(6.94 \mathrm{mg} / \mathrm{mL})$ or physiological saline $(0.9 \% \mathrm{NaCl})$, respectively. All piglets had access to breast milk and water ad libitum and had no other creep feed throughout the experiment period.

At 8:00 am of days 7 and 21 (weaning day), one piglet per group in each litter was randomly selected and then anesthetized and euthanized with a jugular vein injection of $4 \%$ sodium pentobarbital solution (40 mg/kg body weight). Blood samples were taken from the anterior vena cava and centrifuged at $3000 \mathrm{rpm}$ for $15 \mathrm{~min}$, the serum was then stored at $-28^{\circ} \mathrm{C}$ for the analysis of cytokine concentrations. 
Segments of the distal ileum were removed and fixed by immersion in $10 \%(\mathrm{v}: \mathrm{v})$ phosphate buffered formalin for histologic study. The ileal mucosa was collected by scraping with sterile glass microscope slides and rapidly stored at $-80{ }^{\circ} \mathrm{C}$ for further analysis.

The gut microbiota of piglets used in this study have been analyzed in our previous study [19].

\subsection{Morphometric Analysis}

Ileum tissue samples for the morphometric study were dehydrated and embedded in paraffin wax, sectioned at $4 \mu \mathrm{m}$, and stained with hematoxylin and eosin. Villus height and crypt depth were measured by a NIS-Elements F software in a bright field microscope (Nikon, Japan). Villus height was measured from the top of the villus to the top of the lamina propria. Crypt depth was measured from the base upwards to the region of transition between the crypt and villus.

\subsection{Serum Cytokines Analysis}

The concentrations of interferon gamma (IFN- $\gamma$ ), interleukin 1 alpha (IL-1 $\alpha)$, interleukin 1 beta (IL-1 $\beta$ ), interleukin 2 (IL-2), interleukin 4 (IL-4), interleukin 6 (IL-6), interleukin 8 (IL-8), interleukin 10 (IL-10), interleukin 12 (IL-12), and tumor necrosis factor alpha (TNF- $\alpha$ ) in the serum were determined with the ELISA kit (the US China Business Association (USCN), USA), according to the manufacturer's instructions.

\subsection{RNA Isolation and Sequencing}

The RNA was isolated from ileum mucosa with RNAiso Plus Total RNA extraction reagent (Takara) according to the instructions, and the integrity was inspected through an Agilent Bioanalyzer 2100 (Agilent technologies, Santa Clara, CA, USA). Three samples in each group were randomly selected for sequencing for reducing the cost of the experiment. The rRNA of the purified RNA was removed with rRNA removal bead, and then the RNA was divided into fragment and converted to cDNA. The cDNA was purified, and sequencing adaptors were attached to the fragments. Suitable fragments were isolated and amplified by PCR. The libraries outcomes were sequenced by Illumina HiSeq 2500. The average number of reads of samples was 8.54G, and the Q20 ratios were all higher than $90 \%$. Qualified reads were further analyzed. The information of quality control during sequencing is showed in Supplementary Table S1. The preparation and sequencing of libraries was performed by Shanghai Biotechnology Corporation (Shanghai, China).

\subsection{Quantitative Real-Time PCR}

Quantitative analysis real-time PCR was performed on ABI 7300 (Applied Biosystems, Foster City, CA, USA) using SybrGreen (Roche, Switzerland) according to the manufacturer's instructions. The expression of inflammatory cytokines genes, tight junction genes, and intestinal development genes were determined. Besides, the gene expression of C-C motif chemokine ligand 4 (CCL4) [21], C-C motif chemokine ligand 5 (CCL5) [22], C-X-C motif chemokine ligand 9 (CXCL9) [23], CD19 molecule (CD19) [24], inducible T cell costimulator (ICOS) [25], and C-X-C motif chemokine receptor 6 (CXCR6) [26] was determined to validate the result of RNA-sequencing (RNA-seq). The primers used are shown in Supplementary Table S2. The expression of the genes was calculated by normalizing the results to the expression of $\beta$-actin gene with formula $2^{-\Delta \Delta C t}$.

\subsection{Data Analysis}

The data analysis of morphology, quantitative real-time PCR, and serum cytokines was implemented with SPSS (version 20) as a randomized block design. A litter was regarded as an experimental unit, meaning $n=5$. The differences among groups were evaluated using one-way analysis of variance (ANOVA) with $p<0.05$ as the criteria to declare significantly different. 
Regarding the RNA-seq, the raw data were filtered with Seqtk, and then the preprocessed data underwent genome mapping by spliced mapping algorithm of Hisat2 (version: 2.0.4) [27] After comparison, the fragment numbers of each gene were counted with Stringtie (version: 1.3.0) [28,29] and then were normalized by TMM (trimmed mean of M values) [30]. Finally, the fragments per kilobase of exon model per million mapped reads (FPKM) of each gene were calculated using the Perl script. The edgeR [31] was used to analyze the differentially expressed genes among samples and the fold-change (FC) values were calculated based on FPKM. The genes with $p$ value $<0.05$ and FC $>2$ or $<0.5$ are thought to be significant differences and are selected for further analysis.

The gene ontology (GO) enrichment analysis and Kyoto Encyclopedia of Genes and Genomes (KEGG) Pathway enrichment analysis was performed on the Database for Annotation, Visualization and Integrated Discovery (DAVID) Bioinformatics Resources 6.8 (https://david.ncifcrf.gov/tools.jsp, accessed 15 June 2018). The Fisher's exact test was selected as the statistical method, and false discovery rate (FDR) correct method was the FDR value. The difference was seen as significant when $p<0.05$.

\section{Results}

\subsection{Ileal Morphological Structure}

As shown in Table 1, the crypt depth of the ileum both in AM and FMT groups were significantly lower than that in the $\mathrm{CO}$ group on day $7(p<0.05)$. However, no significant difference in ileal morphology was observed in all groups on day 21.

Table 1. Morphology analysis of ileum in neonatal pigs in the amoxicillin (AM), fecal microbiota transplantation (FMT), and control (CO) groups on days 7 and 21.

\begin{tabular}{|c|c|c|c|c|c|c|}
\hline \multirow{2}{*}{ Item } & \multicolumn{3}{|c|}{$7 \mathrm{~d}$} & \multicolumn{3}{|c|}{$21 \mathrm{~d}$} \\
\hline & $\mathrm{CO}$ & AM & FMT & $\mathrm{CO}$ & AM & FMT \\
\hline \multirow{2}{*}{ Villus height, $\mu \mathrm{m}$} & 694.65 & 679.60 & 607.97 & 264.69 & 271.55 & 222.02 \\
\hline & $(66.66)$ & $(26.00)$ & $(48.76)$ & $(3.57)$ & $(8.48)$ & $(44.56)$ \\
\hline \multirow{2}{*}{ Crypt depth, $\mu \mathrm{m}$} & 73.45 & 63.14 & 56.92 & 73.34 & 67.39 & 72.62 \\
\hline & $(1.67)^{\mathrm{a}}$ & $(4.38)^{b}$ & $(0.91)^{b}$ & $(1.18)$ & $(1.01)$ & $(3.81)$ \\
\hline \multirow{2}{*}{ Villus height/Crypt depth } & 9.47 & 10.90 & 10.67 & 3.61 & 4.03 & 3.85 \\
\hline & $(0.95)$ & $(0.57)$ & $(0.77)$ & $(0.06)$ & $(0.13)$ & $(0.53)$ \\
\hline
\end{tabular}

Data were presented as mean (SEM), $n=5$. Mean values within a line with different superscript letters differ significantly $(p<0.05)$. SEM: standard error of mean.

\subsection{Serum Inflammatory Cytokines Concentrations}

On day 7 , there were no changes in concentrations of all detected cytokines among groups. On day 21 , FMT significantly increased the concentration of IL-8 $(p<0.05)$ in plasma compared to the CO group. The concentration of IFN- $\gamma$ was significantly declined $(p<0.05)$ in the AM compared to the FMT and CO. No significant difference in the concentration of IL-2, IL-6, IL-10, IL-12, and TNF $\alpha$ was observed among three groups (Table 2). 
Table 2. Concentration of serum cytokines of piglets in the amoxicillin (AM), fecal microbiota transplantation (FMT), and control (CO) groups on days 7 and 21.

\begin{tabular}{ccccccc}
\hline \multirow{2}{*}{ Items, ng/mL } & \multicolumn{5}{c}{$\mathbf{7 ~ d}$} & \multicolumn{3}{c}{$\mathbf{2 1 ~ d}$} \\
\cline { 2 - 7 } & $\mathbf{A M}$ & FMT & CO & AM & FMT & CO \\
\hline IFN- $\gamma$ & $16.12(1.17)$ & $17.65(0.77)$ & $17.61(1.35)$ & $13.26(0.89)^{\mathrm{b}}$ & $19.35(1.11)^{\mathrm{a}}$ & $17.66(1.55)^{\mathrm{a}}$ \\
IL-2 & $1.27(0.25)$ & $1.05(0.12)$ & $1.35(0.19)$ & $0.86(0.14)$ & $0.80(0.07)$ & $0.92(0.1)$ \\
IL-6 & $0.820(0.14)$ & $0.67(0.12)$ & $0.91(0.1)$ & $0.25(0.051)$ & $0.19(0.04)$ & $0.26(0.05)$ \\
IL-8 & $0.26(0.03)$ & $0.19(0.03)$ & $0.18(0.03)$ & $0.41(0.05)^{\mathrm{ab}}$ & $0.47(0.04)^{\mathrm{a}}$ & $0.34(0.03)^{\mathrm{b}}$ \\
IL-10 & $2.39(0.27)$ & $2.00(0.28)$ & $2.78(0.32)$ & $0.82(0.12)$ & $0.64(0.12)$ & $0.95(0.15)$ \\
IL-12 & $0.46(0.09)$ & $0.44(0.06)$ & $0.55(0.10)$ & $0.77(0.04)$ & $0.74(0.07)$ & $0.76(0.07)$ \\
\hline
\end{tabular}

Data were presented as mean (SEM), $n=5$. Mean values within a line with different superscript letters differ significantly $(p<0.05)$. SEM: standard error of mean. IFN- $\gamma$ : interferon gamma; IL-2: interleukin 2; IL-6: interleukin 6; IL-8: interleukin 8; IL-10: interleukin 10; IL-12: interleukin 12.

\subsection{Expression of Genes Related to Intestinal Epithelial Tight Junction Proteins, Toll-Like Receptors, and Inflammatory Cytokines in the Ileum}

Compared with the CO group, the mRNA expression of ZO-1 was higher $(p<0.05)$ in the AM group on day 7 , while was lower $(p<0.05)$ in the FMT group on day 21. No significant difference in the mRNA expression of occludin was observed among the three groups (Figure 1A). On day 21, the FMT and AM group showed a significant reduction $(p<0.05)$ of toll-like receptor 4 (TLR4) mRNA expression compared to the $\mathrm{CO}$ group. No significant difference in the mRNA expression of toll-like receptor 2 (TLR2) was observed among the three groups (Figure 1B). On day 7, the mRNA expression of pro-inflammatory cytokine IL-8 in the ileum in the AM group was significantly increased $(p<0.05)$ compared to the CO group. On day 21, for pro-inflammatory cytokine TNF- $\alpha$ and anti-inflammatory cytokine TGF- $\beta$, the mRNA expression in the FMT and AM group was significantly lower $(p<0.05)$ than that of the CO group. No significant difference in the mRNA expression of $I L-1 \beta$, IL-10, interferon gamma (IFN- $\gamma$ ), IL-6, IL-18, and histone deacetylase 1 (HDAC1) was observed among the three groups (Figure 1C).

\subsection{Expression of Genes Related to Intestinal Development in the Ileum}

No significant difference was observed in the mRNA expression of the intestinal development-related genes including insulin-like growth factor 1 receptor (IGF-1R), insulin-like growth factor 1 (IGF-1), glucagon-like peptide 2 (GLP-2), and epidermal growth factor (EGF) in the ileum among three groups (Supplementary Figure S1).

\subsection{Transcriptomic Profiling of the Ileal Mucosa}

On day 7, there were 230 differentially expressed genes between AM and $\mathrm{CO}$ groups, 86 between FMT and CO, and 275 between AM and FMT, respectively. On day 21, there were 317 differentially expressed genes between AM and CO groups, 79 between FMT and CO groups, and 201 between AM and FMT (Figure 2).

As show in Figure 3, gene ontology (GO) enrichment analysis were performed. On day 7, a total of 22 terms were significantly affected, and most of them were related to immunity (Figure 3A). On day 21, 39 terms were significantly altered, and most of them were related to the immunity process, material transportation, and transmission of information. Among them, 31 terms were significantly affected by AM (Figure 3B). FMT altered major immune activities from B cell immunity on day 7 to $T$ cell immunity on day 21. Supplementary Table S3 shows the up- or down-regulated GO terms in comparisons among groups. On day 7, GO terms about B cell receptor signaling pathway, regulation of B cell proliferation, and positive regulation of interferon-gamma production were significantly downregulated in $\mathrm{AM} / \mathrm{CO}$ and $\mathrm{AM} / \mathrm{FMT}$, and immune response, inflammatory response, and chemokine-mediated signaling pathway were significantly upregulated in FMT/CO. On day 21, compared with the control 
group, AM significantly upregulated immune response, chemokine-mediated signaling pathway, and lymphocyte chemotaxis, and FMT upregulated positive regulation of cAMP metabolic process, positive regulation of cAMP-mediated signaling, and chemokine-mediated signaling pathway. In AM/FMT, negative regulation of complement activation, classical pathway, leukotriene metabolic process, and transmembrane transport were significantly upregulated, but carbohydrate transmembrane transport was significantly downregulated (Supplementary Table S3).

A

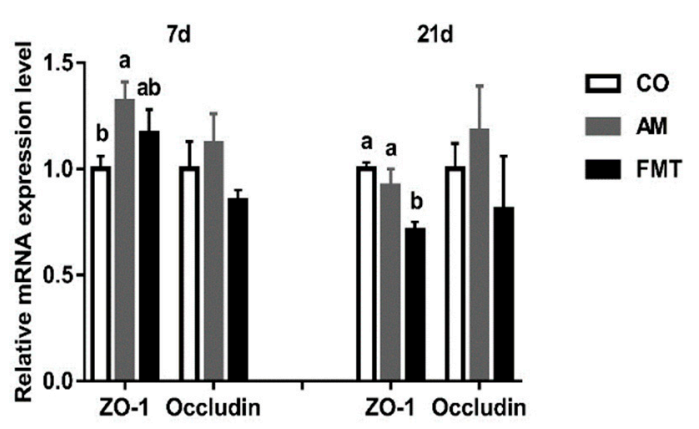

\section{B}

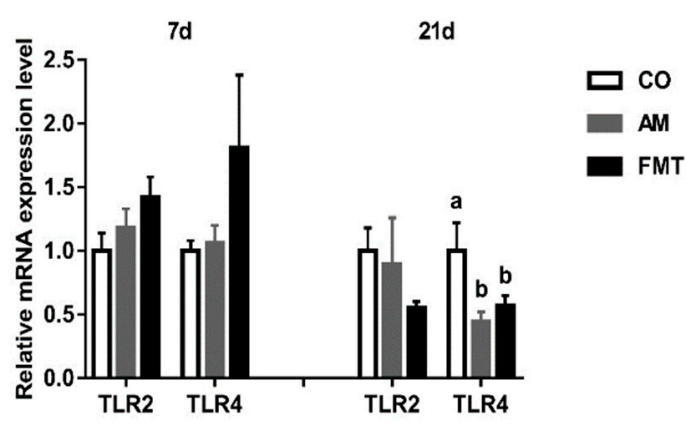

C

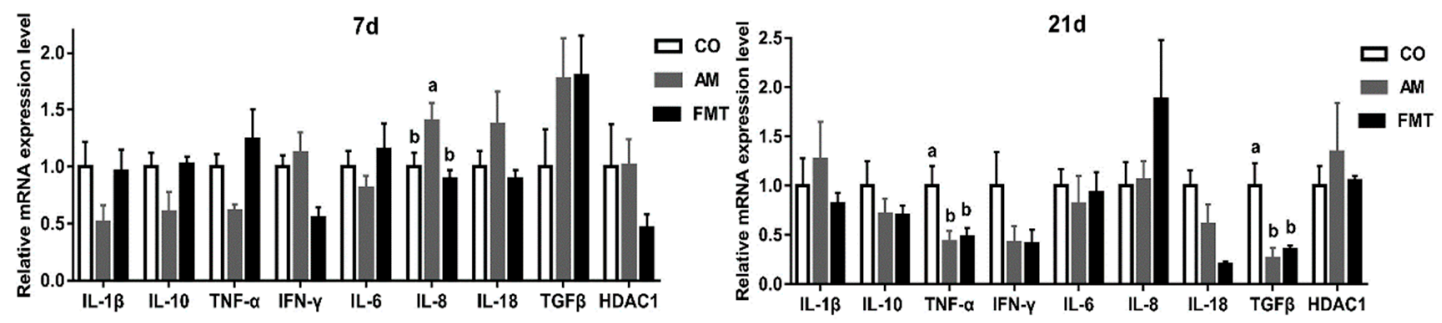

Figure 1. Relative mRNA expression of genes related to intestinal epithelial tight junction proteins (A), toll-like receptors and (B) inflammatory cytokines (C) in the ileum in the amoxicillin (AM), fecal microbiota transplantation (FMT), and control (CO) groups on days 7 and 21. Columns with different letters differ significantly $(p<0.05)$.

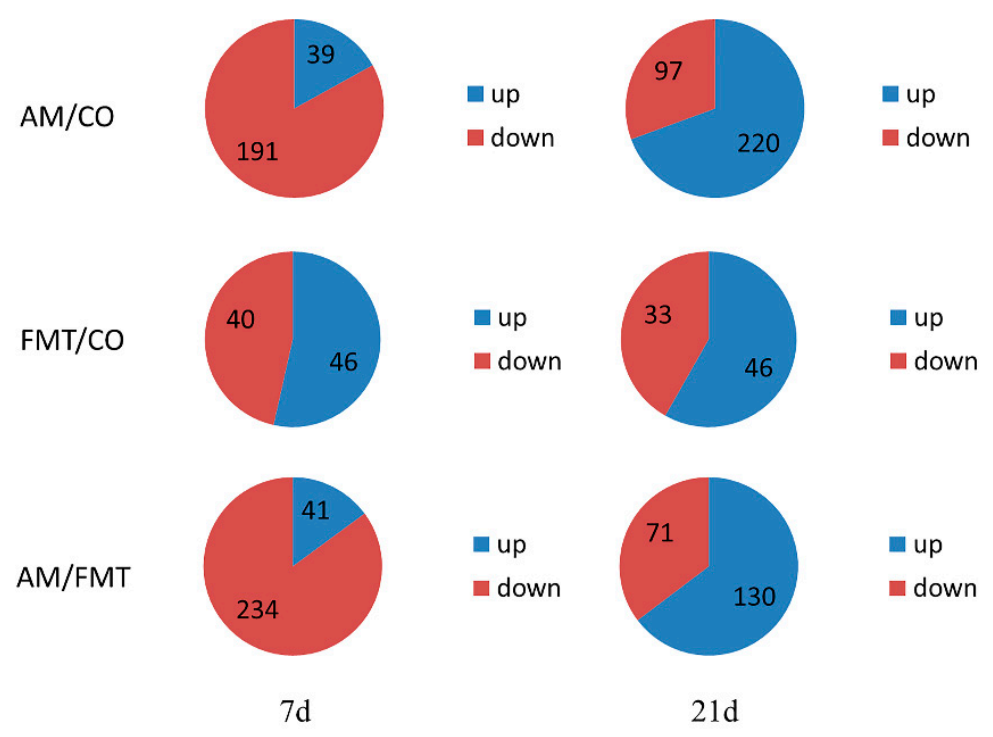

Figure 2. Numbers of the total differentially expressed genes as well as the up- and down-regulated genes in the ileum mucosa of pigs among the amoxicillin (AM), fecal microbiota transplantation (FMT), and control (CO) groups on days 7 and 21. 
A

\begin{tabular}{|c|c|c|c|c|c|}
\hline AM/FMT & FMT/CO & $\mathrm{AM} / \mathrm{CO}$ & Go term ID & Description & \\
\hline & & & GO:0050853 & B cell receptor signaling pathway & \multirow{13}{*}{ immune process } \\
\hline & & & GO:0030888 & regulation of $\mathrm{B}$ cell proliferation & \\
\hline & & & GO:0032729 & positive regulation of interferon-gamma production & \\
\hline & & & GO:0050764 & regulation of phagocytosis & \\
\hline & & & GO:0002467 & germinal center formation & \\
\hline & & & GO:0031663 & lipopolysaccharide-mediated signaling pathway & \\
\hline & & & GO:0006955 & immune response & \\
\hline & & & GO:0006954 & inflammatory response & \\
\hline & & & GO:0070098 & chemokine-mediated signaling pathway & \\
\hline & & & GO:2000503 & positive regulation of natural killer cell chemotaxis & \\
\hline & & & GO:0072678 & T cell migration & \\
\hline & & & GO:0032496 & response to lipopolysaccharide & \\
\hline & & & GO:0002690 & positive regulation of leukocyte chemotaxis & \\
\hline & & & GO:0001504 & neurotransmitter uptake & \multirow{2}{*}{$\begin{array}{l}\text { Transmission of } \\
\text { information }\end{array}$} \\
\hline & & & GO:0000165 & MAPK cascade & \\
\hline & & & GO:0045944 & positive regulation of transcription from RNA polymerase & \multirow{3}{*}{ genetic expression } \\
\hline & & & & II promoter & \\
\hline & & & GO:0042346 & positive regulation of NF-kappaB import into nucleus & \\
\hline & & & GO:0006935 & chemotaxis & \multirow[t]{2}{*}{ cell process } \\
\hline & & & GO:0042127 & regulation of cell proliferation & \\
\hline & & & GO:0009086 & methionine biosynthetic process & metabolic process \\
\hline & & & GO:0060267 & positive regulation of respiratory burst & \multirow[b]{2}{*}{ other } \\
\hline & & & GO:0001974 & blood vessel remodeling & \\
\hline \multirow{2}{*}{$P$-value } & \multirow{2}{*}{\multicolumn{2}{|c|}{0}} & & & \\
\hline & & & & 0.025 & \\
\hline
\end{tabular}

B

\begin{tabular}{|c|c|c|c|c|}
\hline AM/FMT & $\mathrm{FMT} / \mathrm{CO} \quad \mathrm{AM} / \mathrm{CO}$ & Go term ID & Description & \multirow{21}{*}{ immune process } \\
\hline & & GO:0006955 & immune response & \\
\hline & & GO:0070098 & chemokine-mediated signaling pathway & \\
\hline & & GO:0048247 & lymphocyte chemotaxis & \\
\hline & & GO:0071356 & cellular response to tumor necrosis factor & \\
\hline & & GO:0031295 & $T$ cell costimulation & \\
\hline & & GO:0002690 & positive regulation of leukocyte chemotaxis & \\
\hline & & GO:0071347 & cellular response to interleukin-1 & \\
\hline & & GO:0010818 & $\mathrm{T}$ cell chemotaxis & \\
\hline & & GO:0030593 & neutrophil chemotaxis & \\
\hline & & GO:0032496 & response to lipopolysaccharide & \\
\hline & & GO:0002548 & monocyte chemotaxis & \\
\hline & & GO:0006954 & inflammatory response & \\
\hline & & GO:0051607 & defense response to virus & \\
\hline & & GO:0071346 & cellular response to interferon-gamma & \\
\hline & & GO:0071222 & cellular response to lipopolysaccharide & \\
\hline & & GO:0050852 & $\mathrm{T}$ cell receptor signaling pathway & \\
\hline & & GO:0060100 & positive regulation of phagocytosis, engulfment & \\
\hline & & GO:0045959 & negative regulation of complement activation, classical pathwa & \\
\hline & & GO:0010760 & negative regulation of macrophage chemotaxis & \\
\hline & & GO:0090026 & positive regulation of monocyte chemotaxis & \\
\hline & & GO:0055085 & transmembrane transport & \multirow{5}{*}{ Material transportion } \\
\hline & & GO:0051281 & positive regulation of release of sequestered calcium ion into & \\
\hline & & & cytosol & \\
\hline & & GO:0034219 & carbohydrate transmembrane transport & \\
\hline & & GO:0071918 & urea transmembrane transport & \\
\hline & & GO:0043950 & positive regulation of CAMP-mediated signaling & \multirow{4}{*}{$\begin{array}{l}\text { Transmission of } \\
\text { information }\end{array}$} \\
\hline & & GO:0030816 & positive regulation of cAMP metabolic process & \\
\hline & & GO:0050850 & positive regulation of calcium-mediated signaling & \\
\hline & & GO:0070374 & positive regulation of ERK1 and ERK2 cascade & \\
\hline & & GO:0010629 & negative regulation of gene expression & \multirow[t]{2}{*}{ genetic expression } \\
\hline & & GO:0045071 & negative regulation of viral genome replication & \\
\hline & & GO:0060326 & cell chemotaxis & \multirow[t]{2}{*}{ cell process } \\
\hline & & GO:0042127 & regulation of cell proliferation & \\
\hline & & GO:0055114 & oxidation-reduction process & \multirow{5}{*}{ metabolic process } \\
\hline & & GO:0016485 & protein processing & \\
\hline & & GO:0006629 & lipid metabolic process & \\
\hline & & GO:0006691 & leukotriene metabolic process & \\
\hline & & GO:0008152 & metabolic process & \\
\hline & & GO:0070295 & renal water absorption & \multirow[t]{2}{*}{ other } \\
\hline & & GO:0007596 & blood coagulation & \\
\hline
\end{tabular}

Figure 3. Gene ontology (GO) term enrichment analysis of the biological processes of the differentially expressed genes in the ileum mucosa of pigs induced by the amoxicillin (AM), fecal microbiota transplantation (FMT), and control (CO) groups on (A) days 7 and (B) 21 . 
KEGG pathway enrichment is displayed in Figure 4. On day 7, the cytokine-cytokine receptor interaction was significantly affected by FMT. Sphingolipid metabolism, primary immunodeficiency, and the B cell receptor signaling pathway were significantly affected by AM. Compared with FMT, cell adhesion molecules (CAMs), the intestinal immune network for IgA production, the chemokine signaling pathway, primary immunodeficiency, and the cytokine-cytokine receptor interaction were significantly affected by AM (Figure 4A).

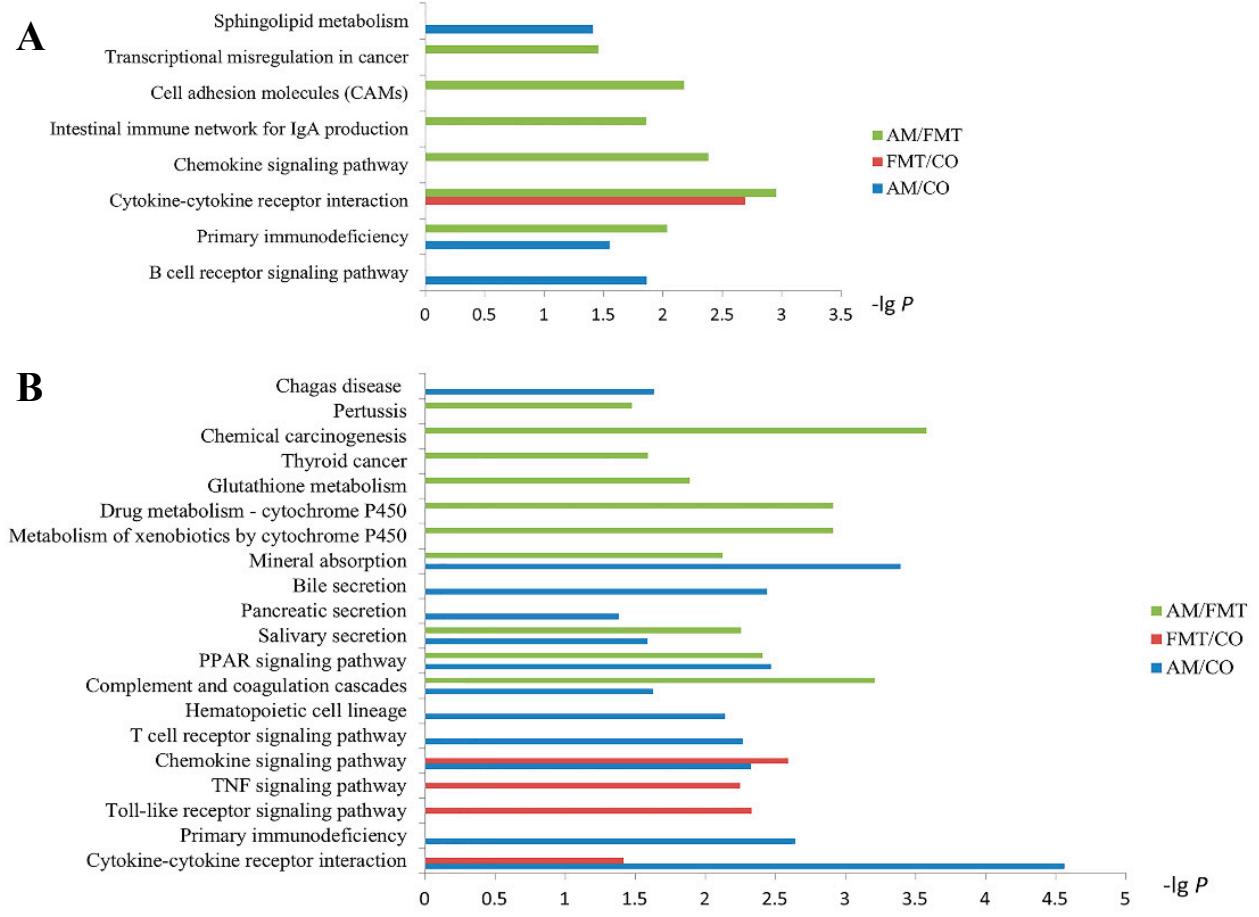

Figure 4. Kyoto Encyclopedia of Genes and Genomes (KEGG) pathway analysis of the differentially expressed genes in the ileum mucosa among the amoxicillin (AM), fecal microbiota transplantation (FMT), and control (CO) groups on (A) days 7 and (B) 21.

On day 21, the cytokine-cytokine receptor interaction and chemokine signaling pathway were significantly affected by AM and FMT. Primary immunodeficiency, the T cell receptor signaling pathway, hematopoietic cell lineage, complement and coagulation cascades, the PPAR signaling pathway, salivary secretion, pancreatic secretion, bile secretion, and mineral absorption were significantly affected by AM. The toll-like receptor signaling pathway, TNF signaling pathway, and graft-versus-host disease (not shown) were significantly affected by FMT. Compared with FMT, glutathione metabolism, metabolism of xenobiotics by cytochrome P450, drug metabolism-cytochrome P450, mineral absorption, salivary secretion, the PPAR signaling pathway, and complement and coagulation cascades were significantly affected by AM (Figure 4B).

\subsection{Validation of RNA-Sequencing by Quantitative Real-Time PCR}

In order to validate the reliability of RNA-seq results, we validated six genes including CCL4, CCL5, CXCL9, CD19, ICOS, and CXCR6 by quantitative real-time PCR (Supplementary Figure S2). The results showed that the expressions of validated genes were consistent with the results of RNA-seq.

\section{Discussion}

In a previous study, we reported the effects of early intervention with amoxicillin and maternal fecal microbiota on gut microbiota and metabolite profiles in neonatal piglets [19]. Here, we investigated the effects of these two early interventions on intestinal function in the ileum of neonatal piglets by 
transcriptome analysis, quantitative real-time PCR analysis, as well as intestinal morphology and serum cytokines analysis. The gene expression landscapes should be a valuable resource for researchers interested in exploring molecular mechanisms for the effects of antibiotic administration on neonatal pig ileum.

\subsection{Effects of Early Maternal Fecal Microbiota and Antibiotics Intervention on Intestinal Morphology and Barrier Function in the Ileum}

The morphology of the intestinal mucosa can reveal some information about gut health. A decrease in crypt depth suggests a reduction in tissue turnover and the surface area for nutrient absorption [32]. In the present study, both amoxicillin and maternal fecal microbiota supplementation decreased crypt depth in the ileum on day 7, which indicated a negative effect on intestinal structure, whereas there was no significant difference on day 21 among the three groups. Similarly, it was reported that the supplementation of antibiotics could decrease colonic crypt depth [33]. In a recent study, treatments of fecal microbiota transplantation from different breeds of swine all exerted no effect on ileal crypt depth in suckling pigs in the long term [34]. Combined with mRNA expression of genes related to intestinal development, these two interventions almost had no effect on ileal development both on days 7 and 21 . Thus, in this study, we consider that early intervention with antibiotic or maternal fecal microbiota has a minimal influence on ileal structure on days 7 and 21.

A complete intestinal barrier can isolate the entry of enteric toxic macromolecules and harmful bacteria and is essential for protecting the gut. The tight junction on the intestinal mucosa is an important part of the intestinal barrier, which prevents the spread of bacteria and other antigens in the epithelium [35]. ZO-1 and occludin, two important tight junction proteins, are commonly used to measure intestinal barrier and permeability [36]. The AM upregulated the mRNA expression of ZO-1 in the ileum on day 7 in piglets, suggesting that the administration of antibiotics may affect intestinal barrier function and the integrity of the intestinal mucosal structure by ZO-1. Toll-like receptors (TLRs) were identified to recognize pathogen ligands and regulate the immune response of the intestinal epithelium by transmitting intestinal bacterial signals [37]. TLR4 is an important transmembrane receptor of LPS, which is responsible for LPS-induced inflammatory cytokines production. On day 21, the decline in TLR4 mRNA expression in the AM group suggested that antibiotics may affect the LPS/TLR4 signal transduction pathway [38].

\subsection{Effects of Early Maternal Fecal Microbiota and Antibiotics Intervention on Transcriptomic Profiles in the Ileum}

Generally speaking, the total numbers of DEGs between day 7 and day 21 are roughly equal in three comparisons (AM/CO, FMT/CO, AM/FMT), but actually, the proportion of upregulated and downregulated genes were significantly altered in AM/CO and AM/FMT. Then, GO and KEGG analyses were performed on DEGs in each comparison. The data showed that, in these comparisons, the number of GO terms and enriched KEGG pathways in a pattern of time effect were greater on day 21 than day 7.

Microbiota colonization after birth is the most important trigger for immune system development [39] and early modulation on microbiota is bound to modify the future immune phenotype of the host [2]. Ileum is considered as the primary site where the gut mucosal immunity is generated, and that is why we focus on its transcriptomic profile. In our previous study, amoxicillin significantly decreased the relative abundance of pathogenic bacteria Streptococcus and Proteobacteria compared to the control group respectively, on day 7 and 21 in neonatal piglet ileums [19]. Proteobacteria is a microbial signature of dysbiosis in gut microbiota [40]. In our results, almost half of the GO terms were involved in immune functions in the ileum whether on day 7 or 21. Especially in AM/CO, compared with day 7 (GO terms mainly related to B cell immunity, which were downregulated in Supplementary Table S3 ), more GO terms of the immune process, mainly including T cell-mediated immune responses and chemotaxis of immunocytes, were altered predominantly in the ileum on day 21. 
Compared with the CO group, AM significantly downregulated GO terms B cell receptor signaling pathway, regulation of $B$ cell proliferation, and positive regulation of interferon-gamma production on day 7 , but immune response, chemokine-mediated signaling pathway, and lymphocyte chemotaxis were upregulated (Supplementary Table S3). It has been reported that antibiotic administration could alter the gene expression profile of the small intestine and increase expression of genes involved in immune functions in the ileum [41], which were consistent with our results. In FMT/CO, also compared with day 7, GO terms related to the immune process did not vary greatly in the ileum on day 21, primarily involving inflammatory response and chemotaxis of immunocytes. It also has been reported that early intervention with fecal microbiota transplantation could permanently change levels of systemic regulatory $\mathrm{T}$ cells and cytokine production [2].

According to results of KEGG pathway analysis, we observed that the major significant pathways were related to chemokine-mediated pathways both in $\mathrm{AM} / \mathrm{CO}$ and FMT/CO on day 21, and interestingly, almost all expression of chemokine DEGs were upregulated, such as $C$ - $C$ motif chemokine ligand 5 (CCL5), C-X-C motif chemokine ligand 9 (CXCL9), and C-X-C motif chemokine ligand 11 (CXCL11). Furthermore, we looked into the DEGs (including CCL4, CCL5, CXCL9, and CXCL11) enriched in the toll-like receptor signaling pathway in $\mathrm{AM} / \mathrm{CO}$ and FMT/CO on day 21 (Table 3), and tried to interpret the effects of DEGs on intestinal function. Studies have shown that the upregulated genes (CCL4, CCL5) enhanced the chemotaxis of NK cells [42], and CXCL9 and CXCL11 strengthened T cell chemotaxis [43]. Consequently, compared with the CO group, both AM and FMT groups enhanced the expression of inflammatory factors in the ileum on day 21. But it was unclear whether these impacts were good or bad for the host health according to the data we had so far. T cells play an important role in the autoimmune response, and ZAP70 is a critical cytoplasmic tyrosine kinase involved in multiple T-cell receptor signaling pathways [44,45]. In AM/CO, the mRNA expression of ZAP70, CD8B, CD3G, and $C D 3 D$ were all upregulated in the $\mathrm{T}$ cell receptor signaling pathway (Table 3 ), indicating the activation of the T cell [46].

Table 3. Expression of genes related to the toll-like receptor signaling pathway and the $\mathrm{T}$ cell receptor signaling pathway in the ileum mucosa of piglets in the amoxicillin (AM), fecal microbiota transplantation (FMT), and control (CO) groups on days 7 and 21.

\begin{tabular}{ccccccc}
\hline \multirow{2}{*}{ Gene } & \multicolumn{3}{c}{$\mathbf{7} \mathbf{d}$} & & $\mathbf{2 1} \mathbf{d}$ & \\
\cline { 2 - 7 } & $\mathbf{A M} / \mathbf{C O}$ & $\mathbf{F M T / C O}$ & $\mathbf{A M} / \mathbf{F M T}$ & $\mathbf{A M} / \mathbf{C O}$ & $\mathbf{F M T / C O}$ & $\mathbf{A M} / \mathbf{F M T}$ \\
\hline CCL4 & NO & NO & DOWN & UP & NO & NO \\
CCL5 & NO & NO & NO & UP & UP & NO \\
CXCL9 & NO & UP & NO & UP & UP & NO \\
CXCL11 & NO & NO & NO & UP & UP & NO \\
ZAP70 & NO & NO & NO & UP & NO & NO \\
CD8B & NO & NO & NO & UP & NO & NO \\
CD3G & NO & NO & NO & UP & UP & NO \\
CD3D & NO & NO & NO & UP & NO & NO \\
\hline
\end{tabular}

UP: upregulated; DOWN: downregulated; NO: no significant change or $0.5<$ fold change $<2$; CCL4: C-C motif chemokine ligand 4; CCL5: C-C motif chemokine ligand 5; CXCL9: C-X-C motif chemokine ligand 9; CXCL11: C-X-C motif chemokine ligand 11; ZAP70: tyrosine-protein kinase ZAP-70; CD8B: CD8b molecule; CD3G: CD3g molecule; CD3D: CD3d molecule.

\section{Conclusions}

This study investigated the effects of two early microbial interventions on intestinal function in ileum in neonatal piglets. The results indicated that early intervention with antibiotics or maternal fecal microbiota significantly altered gene expression profiles in ileum on days 7 and 21, and notably promoted the expression of chemokines involved in the toll-like receptor signaling pathway on day 21 . Moreover, these early interventions to some extent changed intestinal barrier function, although there was a low impact on ileal morphology and development. The data of this study could be a valuable 
reference for the further research on exploring molecular mechanisms for the effects of antibiotic administration on neonatal pig ileum.

Supplementary Materials: The following are available online at http://www.mdpi.com/2079-6382/9/1/35/s1, Figure S1: Effects of early intervention with maternal fecal microbiota and antibiotics on intestinal development gene of the ileum mucosa among the amoxicillin (AM), fecal microbiota transplantation (FMT) and control (CO) groups on days 7 and 21, Figure S2: The qPCR validation of the RNA-seq. The results are displayed as the values of fold changes in the CO group on days 7 and 21 , and the qPCR data are presented as the means $\pm \operatorname{SEM}(n=5)$. Values with different lowercase letter superscripts indicate a significant difference $(p<0.05)$, and those with the same letter superscripts indicate no significant difference $(p>0.05)$. CO: control; AM: amoxicillin; FMT: fecal microbiota transplantation. CCL4: C-C motif chemokine ligand 4; CCL5: C-C motif chemokine ligand 5; CD19: CD19 molecule; CXCL9: C-X-C motif chemokine ligand 9; CXCR6: C-X-C motif chemokine receptor 6; ICOS: inducible T cell costimulator. Table S1: RNA and sequencing data quality control results, Table S2: Primers lists used for real-time PCR assay in this study, Table S3: The regulated gene ontology (GO) terms in the ileum mucosa of piglets in the amoxicillin (AM), fecal microbiota transplantation (FMT) and control (CO) groups on days 7 and 21 .

Author Contributions: Y.S. conceived the ideas and designed the study. R.X., J.W., and C.L. carried out the experiments. R.X., C.L., J.W., and Y.S. analyzed the data, and R.X., C.L., and Y.S. wrote the manuscript. All authors have read and agreed to the published version of the manuscript.

Funding: This research has received funding from the National Natural Science Foundation of China (31572414 and 31872362).

Conflicts of Interest: The authors declare that the research was conducted in the absence of any commercial or financial relationships that could be construed as a potential conflict of interest.

\section{References}

1. Kamada, N.; Núnez, G. Regulation of the immune system by the resident intestinal bacteria. Gastroenterology 2014, 146, 1477-1488. [CrossRef] [PubMed]

2. Hansen, C.H.F.; Nielsen, D.S.; Kverka, M.; Zakostelska, Z.; Klimesova, K.; Hudcovic, T.; Hansen, A.K. Patterns of early gut colonization shape future immune responses of the host. PLoS ONE 2012, 7, e34043. [CrossRef] [PubMed]

3. Brandtzaeg, P. Development and basic mechanisms of human gut immunity. Nutr. Rev. 1998, 56, S5-S18. [CrossRef] [PubMed]

4. Hooper, L.V.; Wong, M.H.; Thelin, A.; Hansson, L.; Falk, P.G.; Gordon, J.I. Molecular analysis of commensal host-microbial relationships in the intestine. Science 2001, 291, 881-884. [CrossRef] [PubMed]

5. Schokker, D.; Zhang, J.; Zhang, L.L.; Vastenhouw, S.A.; Heilig, H.G.; Smidt, H.; Rebel, J.M.; Smits, M.A. Early-life environmental variation affects intestinal microbiota and immune development in new-born piglets. PLoS ONE 2014, 9, e100040. [CrossRef] [PubMed]

6. Collado, M.C.; Cernada, M.; Baüerl, C.; Vento, M.; Pérez-Martínez, G. Microbial ecology and host-microbiota interactions during early life stages. Gut Microbes 2012, 3, 352-365. [CrossRef] [PubMed]

7. Koenig, J.E.; Spor, A.; Scalfone, N.; Fricker, A.D.; Stombaugh, J.; Knight, R.; Angenent, L.T.; Ley, R.E. Succession of microbial consortia in the developing infant gut microbiome. Proc. Natl. Acad. Sci. USA 2011, 108, 4578-4585. [CrossRef]

8. Bhandari, S.K.; Xu, B.; Nyachoti, C.M.; Giesting, D.W.; Krause, D.O. Evaluation of alternatives to antibiotics using an Escherichia coli $\mathrm{K}^{+} 8^{+}$model of piglet diarrhea: Effects on gut microbial ecology. J. Anim. Sci. 2008, 86, 836-847. [CrossRef]

9. Nathan, C. Antibiotics at the crossroads. Nature 2004, 431, 899-902. [CrossRef]

10. Cho, I.; Yamanishi, S.; Cox, L.; Methé, B.A.; Zavadil, J.; Li, K.; Gao, Z.; Mahana, D.; Raju, K.; Teitler, I.; et al. Antibiotics in early life alter the murine colonic microbiome and adiposity. Nature 2012, 488, 621-626. [CrossRef]

11. Morel, F.B.; Oozeer, R.; Piloquet, H.; Moyon, T.; Pagniez, A.; Knol, J.; Darmaun, D.; Michel, C. Preweaning modulation of intestinal microbiota by oligosaccharides or amoxicillin can contribute to programming of adult microbiota in rats. Nutrition 2015, 31, 515-522. [CrossRef] [PubMed]

12. Antonopoulos, D.A.; Huse, S.M.; Morrison, H.G.; Schmidt, T.M.; Sogin, M.L.; Young, V.B. Reproducible community dynamics of the gastrointestinal microbiota following antibiotic perturbation. Infect. Immun. 2009, 77, 2367-2375. [CrossRef] 
13. Connelly, S.; Subramanian, P.; Hasan, N.A.; Colwell, R.R.; Kaleko, M. Distinct consequences of amoxicillin and ertapenem exposure in the porcine gut microbiome. Anaerobe 2018, 53, 82-93. [CrossRef] [PubMed]

14. Schumann, A. Neonatal antibiotic treatment alters gastrointestinal tract developmental gene expression and intestinal barrier transcriptome. Physiol. Genom. 2005, 23, 235-245. [CrossRef] [PubMed]

15. Khoruts, A.; Sadowsky, M.J. Understanding the mechanisms of fecal microbiota transplantation. Nat. Rev. Gastroenterol. Hepatol. 2016, 13, 508-516. [CrossRef] [PubMed]

16. Quraishi, M.N.; Widlak, M.; Bhala, N.; Moore, D.; Price, M.; Sharma, N.; Iqbal, T.H. Systematic review with meta-analysis: The efficacy of fecal microbiota transplantation for the treatment of recurrent and refractory Clostridium difficile infection. Aliment. Pharm. Ther. 2017, 46, 479-493. [CrossRef]

17. McCormack, U.M.; Curião, T.; Wilkinson, T.; Metzler-Zebeli, B.U.; Reyer, H.; Ryan, T.; Calderon-Diaz, J.A.; Crispie, F.; Cotter, P.D.; Creevey, C.J.; et al. Fecal microbiota transplantation in gestating sows and neonatal offspring alters lifetime intestinal microbiota and growth in offspring. MSystems 2018, 3, e00134-17. [CrossRef]

18. Chen, X.; Xu, J.; Ren, E.; Su, Y.; Zhu, W. Co-occurrence of early gut colonization in neonatal piglets with microbiota in the maternal and surrounding delivery environments. Anaerobe 2018, 49, 30-40. [CrossRef]

19. Lin, C.; Wan, J.; Su, Y.; Zhu, W. Effects of early intervention with maternal fecal microbiota and antibiotics on the gut microbiota and metabolite profiles of piglets. Metabolites 2018, 8, 89. [CrossRef]

20. Hamilton, M.J.; Weingarden, A.R.; Sadowsky, M.J.; Khoruts, A. Standardized frozen preparation for transplantation of fecal microbiota for recurrent clostridium difficile infection. Am. J. Gastroenterol. 2012, 107, 761-767. [CrossRef]

21. Ait-Ali, T.; Díaz, I.; Soldevila, F.; Cano, E.; Li, Y.; Wilson, A.D.; Giotti, B.; Archibald, A.L.; Mateu, L.; Darwich, L. Distinct functional enrichment of transcriptional signatures in pigs with high and low IFN-gamma responses after vaccination with a porcine reproductive and respiratory syndrome virus (PRRSV). Vet. Res. 2017, 47, 104. [CrossRef] [PubMed]

22. Chen, Y.; Jin, H.; Chen, P.; Li, Z.; Meng, X.; Liu, M.; Li, S.; Shi, D.; Xiao, Y.; Wang, X.; et al. Haemophilus parasuis infection activates the NF-kB pathway in PK-15 cells through IкB degradation. Vet. Microbiol. 2012, 160, 259-263. [CrossRef] [PubMed]

23. Ashworth, M.D.; Ross, J.W.; Ritchey, J.W.; Desilva, U.; Stein, D.R.; Geisert, R.D.; White, F.J. Effects of aberrant estrogen on the endometrial transcriptional profile in pigs. Reprod. Toxicol. 2012, 34, 8-15. [CrossRef] [PubMed]

24. Williams, A.R.; Hansen, T.V.A.; Krych, L.; Ahmad, H.F.B.; Nielsen, D.S.; Skovgaard, K.; Thamsborg, S.M. Dietary cinnamaldehyde enhances acquisition of specific antibodies following helminth infection in pigs. Vet. Immunol. Immunopathol. 2017, 189, 43-52. [CrossRef]

25. Okutani, M.; Tsukahara, T.; Kato, Y.; Fukuta, K.; Inoue, R. Gene expression profiles of CD4/CD8 double-positive T cells in porcine peripheral blood. Anim. Sci. J. 2018, 89, 979-987. [CrossRef]

26. Shinkai, H.; Morozumi, T.; Toki, D.; Eguchi-Ogawa, T.; Muneta, Y.; Awata, T.; Uenishi, H. Genomic structure of eight porcine chemokine receptors and intergene sharing of an exon between CCR1 and XCR1. Gene 2005, 349, 55-66. [CrossRef]

27. Kim, D.; Langmead, B.; Salzberg, S.L. HISAT: A fast spliced aligner with low memory requirements. Nat. Methods 2015, 12, 357-360. [CrossRef]

28. Pertea, M.; Pertea, G.M.; Antonescu, C.M.; Chang, T.C.; Mendell, J.T.; Salzberg, S.L. StringTie enables improved reconstruction of a transcriptome from RNA-seq reads. Nat. Biotechnol. 2015, 33, 290-295. [CrossRef]

29. Pertea, M.; Kim, D.; Pertea, G.M.; Leek, J.T.; Salzberg, S.L. Transcript-level expression analysis of RNA-seq experiments with HISAT, StringTie and Ballgown. Nat. Protoc. 2016, 11, 1650-1667. [CrossRef]

30. Robinson, M.D.; Oshlack, A. A scaling normalization method for differential expression analysis of RNA-seq data. Genome Biol. 2010, 11, R25. [CrossRef]

31. Robinson, M.D.; Mccarthy, D.J.; Smyth, G.K. edgeR: A Bioconductor package for differential expression analysis of digital gene expression data. Biogeosciences 2010, 26, 139-140. [CrossRef] [PubMed]

32. Friedman, A.; Bar-Shira, E.; Sklan, D. Ontogeny of gut associated immune competence in the chick. World Poult. Sci. J. 2003, 59, 209-219. [CrossRef]

33. Kroismayr, A.; Sehm, J.; Pfaffl, M.W.; Schedle, K.; Plitzner, C.; Windisch, W. Effects of avilamycin and essential oils on mRNA expression of apoptotic and inflammatory markers, and gut morphology of piglets. Czech J. Anim. Sci. 2008, 53, 377-387. [CrossRef] 
34. Diao, H.; Yan, H.L.; Xiao, Y.; Yu, B.; Zheng, P.; He, J.; Yu, J.; Mao, X.B.; Chen, D.W. Modulation of intestine development by fecal microbiota transplantation in suckling pigs. RSC Adv. 2018, 8, 8709-8720. [CrossRef]

35. Förster, C. Tight junctions and the modulation of barrier function in disease. Histochem. Cell Biol. 2008, 130, 55-70. [CrossRef]

36. Fanning, A.S.; Jameson, B.J.; Jesaitis, L.A.; Anderson, J.M. The tight junction protein ZO-1 establishes a link between the transmembrane protein occludin and the actin cytoskeleton. J. Biol. Chem. 1998, 273, 29745-29753. [CrossRef]

37. Lavelle, E.C.; Murphy, C.; O’Neill, L.A.J.; Creagh, E.M. The role of TLRs, NLRs, and RLRs in mucosal innate immunity and homeostasis. Mucosal Immunol. 2009, 3, 17-28. [CrossRef]

38. Lu, Y.C.; Yeh, W.C.; Ohashi, P.S. LPS/TLR4 signal transduction pathway. Cytokine 2008, 42, 145-151. [CrossRef]

39. Matamoros, S.; Gras-Leguen, C.; Le Vacon, F.; Potel, G.; de La Cochetiere, M.F. Development of intestinal microbiota in infants and its impact on health. Trends Microbiol. 2013, 21, 167-173. [CrossRef]

40. Shin, N.R.; Whon, T.W.; Bae, J.W. Proteobacteria: Microbial signature of dysbiosis in gut microbiota. Trends Biotechnol. 2015, 33, 496-503. [CrossRef]

41. Yu, K.; Mu, C.; Yang, Y.; Su, Y.; Zhu, W. Segment-specific responses of intestinal epithelium transcriptome to in-feed antibiotics in pigs. Physiol. Genom. 2017, 49, 582-591. [CrossRef] [PubMed]

42. Robertson, M.J. Role of chemokines in the biology of natural killer cells. J. Leukoc. Biol. 2002, 71, $173-183$. [PubMed]

43. Cekic, C.; Linden, J. Purinergic regulation of the immune system. Nat. Rev. Immunol. 2016, 16, 177-192. [CrossRef] [PubMed]

44. San Luis, B.; Sondgeroth, B.; Nassar, N.; Carpino, N. Sts-2 is a phosphatase that negatively regulates zeta-associated protein (ZAP)-70 and T cell receptor signaling pathways. J. Biol. Chem. 2011, 286, 15943-15954. [CrossRef] [PubMed]

45. Mege, D.; Di Bartolo, V.; Germain, V.; Tuosto, L.; Michel, F.; Acuto, O. Mutation of tyrosines 492/493 in the kinase domain of ZAP-70 affects multiple T-cell receptor signaling pathways. J. Biol. Chem. 1996, 271, 32644-32652. [CrossRef]

46. Chan, A.C.; Irving, B.A.; Fraser, J.D.; Weiss, A. The zeta chain is associated with a tyrosine kinase and upon T-cell antigen receptor stimulation associates with ZAP-70, a 70-kDa tyrosine phosphoprotein. Proc. Natl. Acad. Sci. USA 1991, 88, 9166-9170. [CrossRef] 